\title{
A GENERALIZATION OF THE HALL SEMIGROUP OF A BAND
}

\author{
MÁRIA B. SZENDREI
}

(Received 31 January 1979; revised 19 February 1980)

Communicated by T. E. Hall

\begin{abstract}
Let $E$ be a band and $\delta$ a compatible partition on it. If $S$ is an orthodox semigroup with band of idempotents $E$ such that there exists a congruence on $S$ inducing the partition $\mathscr{E}$ then we define a homomorphism of $S$ into a Hall semigroup whose kernel is the greatest congruence on $S$ inducing the partition $\mathscr{E}$. On the other hand, we define a subsemigroup $W_{E}^{\delta}$ of the Hall semigroup $W_{E}$ possessing the property that $S$ is an orthodox semigroup with band of idempotents $E$ which has a congruence inducing $E$ if and only if the range of the Hall homomorphism of $S$ into $W_{E}$ is contained in $W_{E}^{f}$.
\end{abstract}

1980 Mathemutics subject classification (Amer. Math. Soc.) : $20 \mathrm{M} 10$.

\section{Introduction}

Two congruence relations on a semigroup containing idempotents are called idempotent-equivalent if they induce the same partition on the set of idempotents. Idempotent-equivalent congruences on inverse semigroups were investigated by Reilly and Scheiblich (1967). Their results were generalized for orthodox semigroups by Meakin (1970). He gave a necessary and sufficient condition for a partition of the band of idempotents $E$ of an orthodox semigroup $S$ to be induced by a congruence relation of $S$ and described the greatest and least congruence relations on $S$ corresponding to such a partition of $E$. Feigenbaum (1976) avoids taking transitive closures from these characterizations. We contribute to these results by generalizing in two directions Hall's theorem providing an orthodox semigroup $W_{E}$ for every band $E$ and a homomorphism $\xi$ of $S$ into $W_{E}$ for every orthodox semigroup $S$ with band of idempotents $E$ such that its kernel is the greatest idempotent separating 
congruence on $S$. If $S$ is an orthodox semigroup with band of idempotents $E$ and $\mathscr{E}$ is a partition of $E$ such that there exists a congruence relation on $S$ inducing the partition $\mathscr{E}$ on $E$ then, in Section 1, we determine a homomorphism of $S$ into a Hall semigroup whose kernel is the greatest congruence on $S$ inducing the partition $\mathscr{E}$ on $E$. As a consequence, we give an easy proof of Feigenbaum's characterization concerning the greatest congruence on $S$ inducing $\mathscr{E}$. In Section 2, for every band $E$ and compatible partition $\mathscr{E}$ on it, we define a full orthodox subsemigroup $W_{E}^{\prime}$ of the Hall semigroup $W_{E}$ with the following properties : (i) $W_{E}^{\prime}$ has a congruence relation inducing the partition $\mathscr{E}$ on $E$, (ii) whenever $S$ is an orthodox semigroup with band of idempotents $E$ and $S$ has a congruence relation which induces the partition $\mathscr{E}$ on $E$ then the range of the Hall homomorphism $\xi$ is contained in $W_{E}^{\delta}$.

Throughout the paper we adopt the terminology and notations of Clifford and Preston (1961, 1967) and Howie (1976). As far as the basic properties of orthodox semigroups are concerned we refer to Howie (1976).

\section{A generalization of the Hall homomorphism}

Let $S$ be an orthodox semigroup with band of idempotents $E$. Suppose $\mathscr{E}=\left\{E_{i}: i \in I\right\}$ is a partition of $E$. Meakin(1970) has shown that $S$ has a congruence relation inducing the partition $\mathscr{E}$ on $E$ if and only if the following conditions are satisfied :

(N1) for every $i, j \in I$ there exists $k \in I$ with $E_{i} E_{j} \subseteq E_{k}$,

(N2) for every pair of elements $s, s^{\prime}$ in $S$ which are inverses of each other and for every $i \in I$ there exists $j \in I$ with $s^{\prime} E_{i} s \subseteq E_{j}$.

He described the greatest and least congruence relations on $S$ inducing the partition $\mathscr{E}$ on $E$ as the transitive closures of certain relations. Feigenbaum (1976) finds characterizations of these congruences in which she avoids taking transitive closures.

REMarks. 1. Condition (N1) is equivalent to requiring that:

(N1)* $E$ is a band $I$ of its subbands $E_{i}(i \in I)$.

2. In condition (N2) it suffices to require that:

(N2)* for every $s \in S$ there exists an inverse $s^{\prime}$ of $s$ such that for every $i \in I$ we have $s^{\prime} E_{i} s \subseteq E_{j}$ for some $j \in I$.

$(\mathrm{N} 2)^{*}$ implies (N2). For if $s^{*}$ is any inverse of $s$ then $s^{*}=\left(s^{*} s\right) s^{\prime}\left(s s^{*}\right)$. Hence if $s^{*} s \in E_{k_{1}}$ and $s s^{*} \in E_{k_{2}}$ then

$$
s^{*} E_{i} s=\left(s^{*} s\right) s^{\prime}\left(s s^{*}\right) E_{i} s \subseteq\left(s^{*} s\right) s^{\prime} E_{k_{2}} E_{i} s .
$$

By condition (N1), we have $E_{k_{2}} E_{i} \subseteq E_{j_{2}}$ for some $j_{2} \in I$ and, by (N2)*, $s^{\prime} E_{j_{2}} s \subseteq E_{j_{1}}$ for some $j_{1} \in I$. Applying (N1) again we obtain an element $j \in I$ with $E_{k_{1}} E_{j_{1}} \subseteq E_{j}$. Thus $s^{*} E_{i} s \subseteq E_{j}$. 
In the sequel by a normal partition we mean a partition satisfying (N1)* and (N2).

In what follows, let $S$ be an orthodox semigroup with band of idempotents $E$ and let $\mathscr{E}=\left\{E_{i}: i \in I\right\}$ be a normal partition of $E$. We generalize the argument in the proof of Howie (1976), Chapter 6 Theorem 2.7 to define a homomorphism of $S$ into a Hall semigroup whose kernel is the greatest congruence on $S$ inducing the partition $\mathscr{E}$ on $E$. This result allows us to prove easily the characterization of this congruence obtained in Feigenbaum (1976).

Define a mapping $\theta_{s^{\prime}, s}:\left(s s^{\prime}\right) l I\left(s s^{\prime}\right) l \rightarrow\left(s^{\prime} s\right) l I\left(s^{\prime} s\right) l$ for every pair of elements $s, s^{\prime} \in S$ which are inverses of each other in the following way : let $i \theta_{s^{\prime}, s}$ be the element $j \in I$ for which $s^{\prime} E_{i} s \subseteq E_{j}$. Clearly, $\theta_{s^{\prime}, s}$ is the uniquely determined mapping making the diagram below commutative :

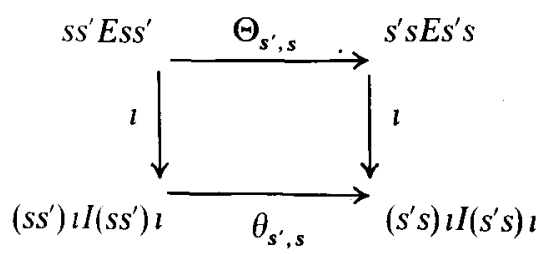

Here $\Theta_{s^{\prime}, s}: s s^{\prime} E s s^{\prime} \rightarrow s^{\prime} s E s^{\prime} s$ is the isomorphism assigning $s^{\prime} e s$ to $e \in s s^{\prime} E s s^{\prime}$. This mapping is used in defining the Hall homomorphism $\xi: S \rightarrow W_{E}$.

LEMMA 1.1. The mapping $\theta_{s^{\prime}, s}$ is an isomorphism of $\left(s s^{\prime}\right) l I\left(s s^{\prime}\right) l$ onto $\left(s^{\prime} s\right) l I\left(s^{\prime} s\right) l$ and $\theta_{s^{\prime}, s}^{-1}=\theta_{s, s^{\prime}}$

The proof of this lemma is immediate.

Now let $i$ be an arbitrary element in $I$. Then

$$
\begin{aligned}
s^{\prime} E_{i} s=s^{\prime}\left(s s^{\prime}\right) E_{i}\left(s s^{\prime}\right) s & \subseteq s^{\prime} E_{\left\langle s s^{\prime}\right) t} E_{i} E_{\left(s s^{\prime}\right) l} s \\
& \subseteq s^{\prime} E_{i \Theta_{\left(s s^{\prime}\right)}} s \subseteq E_{i \Theta_{\left(s s^{\prime}\right),} \theta_{s^{\prime}, s^{\prime}}}
\end{aligned}
$$

where $\Theta_{k}(k \in I)$ is used to denote the mapping of $I$ onto $k I k$ which assigns kik to $i \in I$. Let us define the transformations $\bar{\lambda}_{s}$ and $\bar{\rho}_{s}$ of $I / \mathscr{R}$ and of $I / \mathscr{L}$, respectively, as follows : for every $i \in I$,

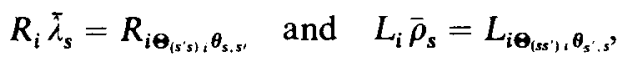

where $s^{\prime}$ is an inverse of $s$. The following lemma shows that $\bar{\lambda}_{s}$ and $\bar{\rho}_{s}$ are well defined.

LEMMA 1.2. Assume that $S$ is an orthodox semigroup with band of idempotents $E$ and $\mathscr{E}=\left\{E_{i}: i \in I\right\}$ is a normal partition of $E$. Let $s \in S$ and $s^{\prime}, s^{*}$ be two inverses of $s$. If $i \mathscr{R} j$ then

$$
i \Theta_{\left(s^{\prime} s\right) \imath} \theta_{s, s^{\prime}} \mathscr{R} j \Theta_{\left(s^{*} s\right)} \theta_{s, s^{*}}
$$


and, similarly, if $i \mathscr{L}_{j}$ then

$$
i \Theta_{\left(s^{\prime}\right) \iota} \theta_{s^{\prime}, s} \mathscr{L}_{j} \Theta_{\left(s^{*}\right) !} \theta_{s^{*}, s^{*}}
$$

ProOf. Since $s^{\prime} s \mathscr{L} s^{*} s$ in $E$ we have $\left(s^{\prime} s\right) l \mathscr{L}\left(s^{*} s\right) \imath$ in $I$. Green's relation $\mathscr{D}$ is a semilattice congruence on $I$ therefore $D_{\left(s^{*} s\right)} l \geqslant D_{\left(s^{\prime}\right) i, j}=D_{i .\left(s^{\prime}\right) t}$ holds in $I$. Thus $i \mathscr{R} j$ implies

$$
\left(s^{\prime} s\right) l \cdot j \cdot\left(s^{*} s\right) l \cdot i .\left(s^{\prime} s\right) l=\left(s^{\prime} s\right) l . j i .\left(s^{\prime} s\right) l=\left(s^{\prime} s\right) i . i .\left(s^{\prime} s\right) i=i \Theta_{\left(s^{\prime} s\right) l} .
$$

Hence we conclude that

$$
\begin{aligned}
\left(s E_{j} s^{*}\right)\left(s E_{i} s^{\prime}\right)=s\left(s^{\prime} s\right) & E_{j}\left(s^{*} s\right) E_{i}\left(s^{\prime} s\right) s^{\prime} \\
& \subseteq s E_{i \boldsymbol{\Theta}_{s} s_{s, h}} s^{\prime} \subseteq E_{i \boldsymbol{\Theta}, \ldots \theta_{s, s}}
\end{aligned}
$$

Hence, by (1), the equality

$$
j \Theta_{\left(s^{*} s\right) t} \theta_{s, s^{*}} . i \Theta_{\left(s^{\prime} s\right) t} \theta_{s, s^{\prime}}=i \Theta_{\left(s^{\prime} s\right) t} \theta_{s, s^{\prime}}
$$

follows. By symmetry,

$$
i \Theta_{\left(s^{\prime} s\right) !} \theta_{s, s^{\prime}} j \Theta_{\left(s^{*} s\right) \iota} \theta_{s, s^{*}}=j \Theta_{\left(s^{*} s\right) l} \theta_{s, s^{*}}
$$

also holds which completes the proof of the first assertion. The second one follows dually.

Now we can state the main result of this section.

THEOREM 1.3. Suppose $S$ is an orthodox semigroup with hand of idempotents $E$ and $\mathscr{E}=\left\{E_{i}: i \in I\right\}$ is a normal partition of $E$. The mapping $\bar{\xi}^{\prime}: S \rightarrow W_{I}$ defined $b y$ $s \bar{\xi}^{*}=\left(\bar{\rho}_{s}, \bar{\lambda}_{s}\right)$, where $\bar{\lambda}_{s}$ and $\bar{\rho}_{s}$ are determined by (2), is a homomorphism. $S \bar{\xi}^{s}$ is a full or thodox subsemigroup of $W_{1}$ and the kernel of $\bar{\xi}^{\prime}$ is the greatest congruence relation on $S$ inducing the partition $\mathscr{E}$ on $E$.

REMARK. If $\mathscr{E}$ is the trivial normal partition of $E$ where $E_{i}(i \in I)$ are singletons then $I$ is actually $E$ and, consequently, $\bar{\lambda}_{s}$ and $\bar{\rho}_{s}(s \in S)$ are just $\lambda_{s}$ and $\rho_{s}$, respectively, used in Howie (1976), Chapter 6 Theorem 2.7, to define the Hall homomorphism $\xi: S \rightarrow W_{E}$. In this case $\xi=\xi^{8}$ and Theorem 1.3 restates this theorem.

ProOf. One can immediately see by (2) that $\left(\bar{\rho}_{s}, \bar{\lambda}_{s}\right) \in W_{l}$. In consequence of (1) and (2) we obtain that $\bar{\xi}^{\delta}$ is a homomorphism. If $e \in E_{\mathrm{k}}$ then $e^{\prime} E_{i} e \subseteq E_{k i k}=E_{i \mathrm{em}}$ for every $i \in I$. Thus, by (2), we have $R_{i} \bar{\lambda}_{e}=R_{i \Theta_{k}}$ and $L_{i} \bar{\rho}_{e}=L_{i \boldsymbol{\theta}_{k^{*}}}$ This means that $e \bar{\xi}^{\phi}=\left(\bar{\rho}_{e}, \bar{\lambda}_{e}\right)=k \psi$ where $\psi: I \rightarrow W_{l}$ is the natural embedding of $I$ in $W_{I}$. This implies, on the one hand, that $S \bar{\xi}^{t}$ contains all the idempotents of $W_{l}$, that is, $S_{\xi} \bar{\xi}^{k}$ is a full orthodox subsemigroup of $W_{l}$. On the other hand, we infer that the congruence $\bar{\xi}^{\ell} \cdot\left(\bar{\zeta}^{E}\right)^{-1}$ induces the partition $\mathscr{E}$ on $E$. Consider an arbitrary congruence $\sigma$ on $S$ 
which induces $\mathscr{E}$ on $E$. Then an isomorphism can be defined between $I$ and the band of idempotents of the factor semigroup $S / \sigma$ by assigning the $\sigma$-class containing $E_{i}$ to $i \in I$. Let us identify the band of idempotents of $S / \sigma$ with $I$ by this isomorphism. If $s$ and $s^{\prime}$ are inverses of each other in $S$ then $s \sigma$ and $s^{\prime} \sigma$ are inverses of each other in $S / \sigma$. Furthermore, if $s^{\prime} E_{i} s \subseteq E_{j}$ then $s^{\prime} \sigma . i . s \sigma=j$ holds in $S / \sigma$. Thus there is no difficulty in verifying that $\xi^{\xi}=\sigma^{\natural} \xi$ where $\xi: S / \sigma \rightarrow W_{I}$ is the Hall homomorphism of $S / \sigma$ into $W_{l}$. Consequently, we have $\sigma \subseteq \bar{\xi}^{\delta} \circ\left(\xi^{\delta}\right)^{-1}$ which proves that $\bar{\xi}^{\delta} \circ\left(\bar{\xi}^{\delta}\right)^{-1}$ is the greatest congruence on $S$ inducing the partition $\mathscr{E}$ on $E$.

REMARK. The last statement of Theorem 1.3 can be proved by observing that the relation $\zeta$ defined in Meakin (1970), Theorem 2.3, is contained in $\bar{\xi}^{\xi} \circ\left(\bar{\xi}^{\xi}\right)^{-1}$. Then the same holds for its transitive closure $\zeta^{t}$. Since $\zeta^{\delta} \circ\left(\xi^{\delta}\right)^{-1}$ induces the partition $\mathscr{E}$ on $E$, Meakin (1970), Theorem 2.3, implies that $\bar{\xi}^{\delta} \circ\left(\bar{\xi}^{f}\right)^{-1}=\zeta^{t}$ and $\zeta^{t}$ is the greatest congruence on $S$ inducing $\mathscr{E}$.

Now we deduce Feigenbaum (1976), Theorem 4.2, from our Theorem 1.3.

COROLlary 1.4. Let $S$ be an orthodox semigroup with band of idempotents $E$. Assume that $\mathscr{E}=\left\{E_{i}: i \in I\right\}$ is a normal partition of $E$. Then the relation

$$
\begin{aligned}
& \zeta^{*}=\left\{(s, t) \in S \times S \text { : there exist inverses } s^{\prime} \text { and } t^{\prime} \text { of } s \text { and } t\right. \text {, respectively, } \\
& \text { such that } i \in I \text { implies } s E_{i} s^{\prime} \subseteq E_{j}, t E_{i} t^{\prime} \subseteq E_{\bar{j}} \text { and } \\
& s^{\prime} E_{i} s \subseteq E_{k}, t^{\prime} E_{i} t \subseteq E_{\vec{k}} \text { for some } j, \vec{\jmath}, k, k \in I \\
& \text { with } j \mathscr{R} \bar{j} \text { and } k \mathscr{L} \bar{k}\}
\end{aligned}
$$

is the greatest congruence on $S$ which induces the partition $\mathscr{E}$ on $E$.

Proof. According to the definition of the homomorphism $\bar{\xi}^{\xi}$ we have $s\left(\xi^{\prime} \circ\left(\xi^{\prime}\right)^{-1}\right) t$ if and only if the following is valid for each pair of inverses $s^{\prime}$ and $t^{\prime}$ of $s$ and $t$, respectively : $i_{1} \mathscr{R} j_{1}$ provided $i \mathscr{R} j, s E_{i} s^{\prime} \subseteq E_{i_{1}}$ and $t E_{j} t^{\prime} \subseteq E_{j_{1}}$, and, dually, $i_{1} \mathscr{L} j_{1}$ provided $i \mathscr{L} j, s^{\prime} E_{i} s \subseteq E_{i_{1}}$ and $t^{\prime} E_{j} t \subseteq E_{j_{1}}$. Lemma 1.2 implies $\zeta^{*} \subseteq \bar{\zeta}^{t} \circ\left(\bar{\zeta}^{\prime}\right)^{-1}$. The reverse inclusion is obvious. The proof is complete by Theorem 1.3 .

\section{The subsemigroup $W_{E}^{\ell}$ of $W_{E}$}

Let $E$ be a band and $\mathscr{E}$ a compatible partition of $E$. In this section we define a full orthodox subsemigroup $W_{E}^{\delta}$ of the Hall semigroup $W_{E}$ which plays just the same role among the orthodox semigroups with band of idempotents $E$ possessing a congruence relation which induces the partition $\mathscr{E}$ on $E$ as $W_{E}$ plays among all orthodox semigroups with band of idempotents $E$. That means, $W_{E}^{g}$ is an orthodox semigroup with band of idempotents $E^{*}$ isomorphic to $E$ which has a congruence 
inducing that partition on $E^{*}$ which corresponds to $\mathscr{E}$ under the natural embedding $\psi: E \rightarrow W_{E}$. Moreover, if $S$ is an orthodox semigroup with band of idempotents $E$ which has a congruence relation inducing $\mathscr{E}$ on $E$ then the Hall homomorphism $\xi$ maps $S$ into $W_{E}^{\prime}$.

Let $E$ be a band. Denote by $\mathscr{U}$ the relation on $E$ for which $e \mathscr{U} f$ if and only if $e E e$ is isomorphic to $f E f$. Suppose $\mathscr{E}=\left\{E_{i}: i \in I\right\}$ is a partition fulfilling the condition (N1)*. The equivalence relation corresponding to the partition $\mathscr{E}$ is denoted by $\pi_{f}$. Clearly, $\pi_{f}$ is a congruence relation on $E$. Denote by $W_{e, f}^{\delta}$ the set of onto isomorphisms $\alpha: e E e \rightarrow f E f$ with the following property : for every $x, y \in e E e, x \pi_{\delta} y$ if and only if $x \alpha \pi_{,} y \alpha$. Consider the following subset of the Hall semigroup $W_{E}$ :

$$
W_{E}^{\prime}=\left\{\left(\rho_{e} \alpha_{l}, \lambda_{f} \alpha_{r}^{-1}\right): e \mathscr{U} f \text { and } \alpha \in W_{e, f}^{\prime}\right\} .
$$

We shall show that $W_{E}^{\prime}$ is an orthodox semigroup and it has the properties stated above. In the proof we need the following lemma concerning the Hall semigroup $W_{E}$ (see the proof of Howie (1976), Chapter 6, Theorem 2.17).

LEMMA 2.1. We are given four elements, $e, f, g$ and $h$ in the band $E$ such that $e$ Ul $f$ and $g$ Ul h. Let $\alpha \in W_{e, f}$ and $\beta \in W_{g, h}$. Then (i) the product

$$
\left(\rho_{e} \alpha_{l}, \lambda_{f} \alpha_{r}^{-1}\right)\left(\rho_{g} \beta_{l}, \lambda_{h} \beta_{r}^{-1}\right)
$$

of two elements in $W_{E}$ equals $\left(\rho_{k} \gamma_{l}, \lambda_{m} \gamma_{r}^{-1}\right)$ where $k=(f g f) \alpha^{-1}, m=(g f g) \beta$ and

$$
\gamma=(\alpha \mid k E k)\left(\Theta_{g f g} \mid f g f E f g f\right)(\beta \mid g f g E g f g) \in W_{k, m} ;
$$

(ii) $\left(\rho_{f} \alpha_{l}^{-1}, \lambda_{e} \alpha_{r}\right)$ is an inverse of the element $\left(\rho_{e} \alpha_{l}, \lambda_{f} \alpha_{r}^{-1}\right)$; and (iii) every idempotent in $W_{E}$ is of the form $\left(\rho_{e}, \lambda_{e}\right)$ for some e in $E$.

LEMMA 2.2. $W_{E}^{\delta}$ is a full orthodox subsemigroup of $W_{E}$.

Proof. If $e \mathscr{U} f$ in $E$ and $\alpha \in W_{e, f}^{\ell}$ then we can easily see that $\alpha^{-1} \in W_{f, e}^{\delta}$. Thus Lemma 2.1(ii) gives an inverse of each element of $W_{E}^{\delta}$ which is also contained in $W_{E}^{\delta}$. If $e^{\mathscr{U} f} f$ and $g \mathscr{U} h$ in $E$ and $\alpha \in W_{e, f}^{s}, \beta \in W_{g, h}^{s}$ then we clearly have $(\alpha \mid k E k) \in W_{k, f g f}^{\prime}$ and $(\beta \mid g f g E g f g) \in W_{g f g, m}^{\ell}$ where $k=(f g f) \alpha^{-1}$ and $m=(g f g) \beta$. Since the partition $\mathscr{E}$ satisfies condition $(\mathrm{N} 1)^{*}$, one can immediately see that $\left(\Theta_{g f g} \mid f g f E f g f\right) \in W_{f g f, g f g}^{s}$. Thus we obtain that their product $\gamma$ defined in Lemma 2.1 is contained in $W_{k, m}^{s}$. Referring to Lemma 2.1(i) we conclude that $W_{E}^{\delta}$ is a subsemigroup of $W_{E}$. It is obvious by Lemma 2.1(iii) that $W_{E}^{\delta}$ contains all the idempotents of $W_{E}$ as the identity automorphism of $e E e(e \in E)$ belongs to $W_{e, e}^{\prime}$. Thus we have shown that $W_{E}^{\delta}$ is a full orthodox subsemigroup of $W_{E}$.

Define a mapping $v^{\delta}: W_{E}^{\delta} \rightarrow W_{E / \pi_{\delta}}$ in the following way. Let $e \in E_{i}$ and $f \in E_{j}$. Suppose $e \mathscr{U} f$ and $\alpha \in W_{e, f}^{s}$. Then the mapping $\bar{\alpha}: E_{i}\left(E / \pi_{g}\right) E_{i} \rightarrow E_{j}\left(E / \pi_{g}\right) E_{j}$ which 
assigns the $\pi_{s}$-class containing $E_{k} \alpha$ to $E_{k}$ for every $k \leqslant i$ is an onto isomorphism. Let

$$
\left(\rho_{e} \alpha_{l}, \hat{\lambda}_{f} \alpha_{r}^{-1}\right) v^{A}=\left(\rho_{E_{i}} \bar{\alpha}_{l}, \lambda_{E_{j}} \bar{\alpha}_{r}^{-1}\right) .
$$

LEMMA 2.3. The mapping $v^{\delta}: W_{E}^{\delta} \rightarrow W_{E / \pi s}$ is a homomorphism whose kernel induces the partition $\mathscr{E}^{*}=\left\{E_{i} \psi: i \in I\right\}$ on the band of idempotents.

ProOF. Let $e \in E_{i}, f \in E_{j}, g \in E_{u}, h \in E_{v}$ such that $e \mathscr{U} f$ and $g \mathscr{U} h$ and let $\alpha \in W_{e, f}^{\delta}$, $\beta \in W_{g, k}^{\delta}$. Then $\bar{\alpha} \in W_{E_{i}, E_{j}}$ and $\bar{\beta} \in W_{E_{y}, E_{v}}$. Suppose $k=(f g f) \alpha^{-1} \in E_{x}$ and $m=(g f g) \beta \in E_{y}$. Clearly, $E_{x}=E_{j u j} \bar{\alpha}^{-1}$ and $E_{y}=E_{u j u} \bar{\beta}$. Moreover, one can see immediately that

$$
\begin{aligned}
\bar{\gamma} & =\overline{(\alpha \mid k E k)\left(\Theta_{g f g} \mid f g f E f g f\right)(\beta \mid g f g E g f g)} \\
& =\overline{(\alpha \mid k E k)}\left(\overline{\left(\Theta_{q f q} \mid f g f E f g f\right.}\right) \overline{(\beta \mid g f g E g f g)} \\
& =\left(\bar{\alpha} \mid E_{x}\left(E / \pi_{\ell}\right) E_{x}\right)\left(\Theta_{E_{u j u}} \mid E_{j u j}\left(E / \pi_{g}\right) E_{j u j}\right)\left(\bar{\beta} \mid E_{u j u}\left(E / \pi_{f}\right) E_{u j u}\right) .
\end{aligned}
$$

Hence we obtain by Lemma 2.1(i) that

$$
\left(\rho_{e} \alpha_{l}, \lambda_{f} \alpha_{r}^{-1}\right) v^{\delta}\left(\rho_{g} \beta_{l}, \lambda_{h} \beta_{r}^{-1}\right) v^{\delta}=\left(\rho_{k} \gamma_{l}, \lambda_{m} \gamma_{r}^{-1}\right) v^{\delta},
$$

that is, $v^{\ell}$ is a homomorphism. Since we have $\left(\rho_{e}, \lambda_{e}\right) v^{\delta}=\left(\rho_{E_{i}}, \lambda_{E_{i}}\right)$ for every idempotent $e \psi=\left(\rho_{e}, \lambda_{e}\right)$ in $W_{E}^{\delta}$ therefore $v^{\mathscr{E}} \circ\left(v^{\delta}\right)^{-1}$ induces the partition $\mathscr{E}^{*}$. The proof is complete.

Now we can state the main result of this section.

THEOREM 2.4. Suppose $E$ is $a$ band and $\mathscr{E}=\left\{E_{i}: i \in I\right\}$ is a partition of $E$ satisfying condition (N1)*. Then $W_{E}^{\delta}$ defined in (3) is a full orthodox subsemigroup of $W_{E}$ and the mapping $v^{\prime}: W_{E}^{s} \rightarrow W_{E / \pi_{g}}$ determined by (4) is a homomorphism whose kernel induces the partition $\mathscr{E}^{*}=\left\{E_{i} \psi: i \in I\right\}$ on the band of idempotents. Furthermore, whenever $S$ and $S^{\prime}$ are orthodox semigroups with bands of idempotents $E$ and $E / \pi_{\xi}$, respectively, and $\varphi: S \rightarrow S^{\prime}$ is a homomorphism with $\varphi \mid E=\pi_{g}^{\natural}$ then $S \xi \subseteq W_{E}^{\xi}$ and $\varphi \xi^{\prime}=\xi v^{\prime}=\xi^{\prime}$. Here $\xi$ and $\xi^{\prime}$ are

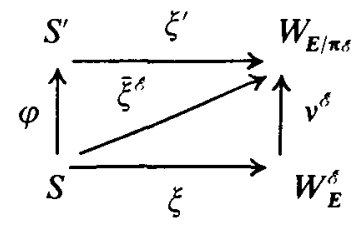

the Hall homomorphisms of $S$ and $S^{\prime}$, respectively.

Proof. By Lemmas 2.2 and 2.3, all we have to prove is the last statement. Assume that $S, S^{\prime}$ and $\varphi$ fulfil the conditions of the theorem. Then $\varphi \circ \varphi^{-1}$ induces the 
partition $\mathscr{E}$ on $E$. Let $s$ and $s^{\prime}$ be inverses of each other in $S$. It is well known that

$$
\Theta_{s^{\prime}, s}: s s^{\prime} E s s^{\prime} \rightarrow s^{\prime} s E s^{\prime} s, \quad e \Theta_{s^{\prime}, s}=s^{\prime} e s
$$

is contained in $W_{s s^{\prime}, s^{\prime} s^{\prime}}$ If $x, y \in s s^{\prime} E s s^{\prime}$ and $x \pi_{f} y$ then $x \Theta_{s^{\prime}, s}=s^{\prime} x s \pi_{f} s^{\prime} y s=y \Theta_{s^{\prime}, s}$ by (N2). The same is valid for the isomorphism $\Theta_{s, s^{\prime}}$. Since they are inverses of each other we infer that $x \pi_{\delta} y$ if and only if $x \Theta_{s^{\prime}, s} \pi_{f} y \Theta_{s^{\prime}, s^{\prime}}$. Thus $\Theta_{s^{\prime}, s} \in W_{s s^{\prime}, s^{\prime} s^{\prime}}$. By the definition of the homomorphism $\xi: S \rightarrow W_{E}$, hence it follows that $S \xi \subseteq W_{E}^{c}$. The equalities $\varphi \xi^{\prime}=\bar{\xi}^{\prime}$ and $\xi v^{\prime}=\bar{\xi}^{\prime \prime}$ are immediate consequences of the definitions of $\xi^{\prime}$ and $v^{t}$. The proof of the theorem is complete.

The author expresses her thanks to the referee for calling her attention to Feigenbaum's paper.

\section{References}

A. H. Clifford and G. B. Preston (1961, 1967), The algebraic theory of semigroups, Volumes I, II (Math. Surveys 7, Amer. Math. Soc., Providence, R.I.).

R. Feigenbaum (1976), 'Kernels of orthodox semigroup homomorphisms', J. Austral. Math. Soc. 22 , 234-245.

J. M. Howie (1976), An introduction to semigroup theory (Academic Press, London, New York, San Francisco).

J. Meakin (1970), 'Idempotent-equivalent congruences on orthodox semigroups', J. Austal. Math. Soc. 11. 221-241.

N. R. Reilly and H. E. Scheiblich (1967), 'Congruences on regular semigroups', Pacific J. Math. 23, $349-360$.

József Attila University

Bolyai Institute

H-6720 Szeged

Aradi vértanúk tere 1

Hungary 